\title{
Arterial Stiffness as a Risk Factor for Subclinical Coronary Artery Calcification in Predialysis Chronic Kidney Disease: From the KNOW-CKD Study
}

\author{
Young Youl Hyun ${ }^{a}$ Hyang Kim ${ }^{a}$ Kook-Hwan Oh ${ }^{b}$ Curie Ahn ${ }^{b}$ Sue K. Park \\ Dong Wan Chae ${ }^{d}$ Seung Hyeok Han ${ }^{e}$ Yong-Soo Kim ${ }^{f}$ Sung Woo Lee ${ }^{g}$ \\ Chang Seong Kim ${ }^{\mathrm{h}}$ Kyu-Beck Lee ${ }^{\mathrm{a}}$ \\ aDepartment of Internal Medicine, Sungkyunkwan University School of Medicine, \\ Kangbuk Samsung Hospital, Seoul, Republic of Korea; ${ }^{b}$ Department of Internal Medicine, \\ Seoul National University College of Medicine, Seoul, Republic of Korea; ${ }^{~}$ Department of \\ Preventive Medicine, Seoul National University College of Medicine, Seoul, Republic of \\ Korea; ${ }^{d}$ Department of Internal Medicine, Seoul National University Bundang Hospital,

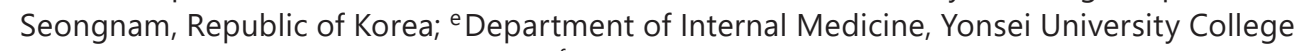

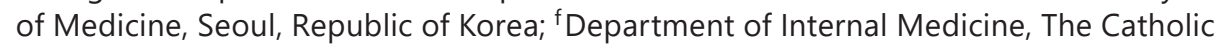 \\ University of Korea, Seoul St. Mary's Hospital, Seoul, Republic of Korea; 9 Department \\ of Internal Medicine, Eulji Medical Center, Eulji University, Seoul, Republic of Korea; \\ hDepartment of Internal Medicine, Chonnam National University Medical School, Gwangju, \\ Republic of Korea
}

\section{Keywords}

Arterial stiffness - Pulse wave velocity · Coronary calcification · Vascular calcification . Chronic kidney disease

\section{Abstract}

Background/Aims: Both arterial stiffness and coronary artery calcification (CAC) are important predictors of cardiovascular disease in the general population and in chronic kidney disease (CKD) patients. Recent studies on arterial stiffness and CAC in subjects with preserved renal function have verified the association between the two. However, the relationship is not well evaluated in CKD patients. Methods: This cross-sectional study analyzed 1,385 predialysis CKD patients from the KNOW-CKD cohort. Participants were divided into four groups according to brachial-ankle pulse wave velocity (baPWV) quartile. Coronary artery calcium scores (CACS) were assessed using cardiac computed tomography and CAC was defined as a CACS 


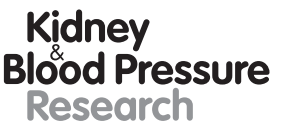

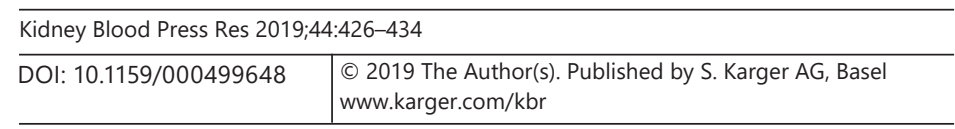

Hyun et al.: Arterial Stiffness and Coronary Calcification

$>100$. Results: CAC prevalence was higher in the higher baPWV groups $(6.4,9.8,23.7$, and $43.8 \%$ for the 1 st to 4 th quartiles of baPWV, respectively, $p<0.001)$. In Tobit regression analyses that were fully adjusted for traditional and renal cardiovascular risk factors, the CACS ratio comparing the highest and lowest baPWV quartiles was $3.03(95 \% \mathrm{Cl}, 1.59-6.87)$. Similarly, the OR for CAC in the highest baPWV quartile compared to the lowest quartile was $1.98(95 \% \mathrm{Cl}$, 1.09-3.60) in a fully adjusted multivariate logistic model. Results were consistent across analyses with different cutoffs for CAC or with different clinically relevant subgroups. Conclusion: Increased arterial stiffness measured by high baPWV was associated with CAC in a predialysis CKD cohort. Longitudinal studies are needed to determine the effect of arterial stiffness on the development or progression of CAC in CKD.

\section{Introduction}

Cardiovascular disease (CVD) is a major cause of death in patients with chronic kidney disease (CKD) [1]. Therefore, prevention and early detection of cardiovascular (CV) complications are critical for successful treatment of CKD patients and for slowing renal function deterioration. Arterial stiffness and vascular calcification are important predictors of CVD in the general population and in CKD patients.

Arterial stiffness refers to the loss of ability of arteries to accommodate stroke volume [2]. It is well known that arterial stiffness increases with decreasing renal function [3], which is thought to be the result of complex actions, such as alterations in the extracellular matrix, advanced glycation end products, endothelial dysfunction, chronic inflammation, and vascular calcification [4]. Importantly, an increase in arterial stiffness is associated with a rapid decline in renal function [5] and high incidence of CVD and CV mortality [6, 7].

Calcification is another commonly observed vascular alteration in CKD patients that is broadly divided into the following categories: atherosclerotic intimal calcification, arteriosclerotic medial calcification, cardiac valvular calcification, and calciphylaxis [8]; all of which are poor prognostic factors in CKD patients $[9,10]$. Coronary artery calcification (CAC) was recently established as a predictor of $\mathrm{CV}$ complications in the general population and is recommended for use in assessing CV risk in asymptomatic adults with intermediate risk [11]. Recent studies have identified CAC as a predictor of CV disease and mortality in CKD patients, suggesting the possibility of a risk prediction tool for CKD [12,13].

Arterial stiffness can be easily measured by PWV (pulse wave velocity), and recent studies of PWV and CAC in subjects with preserved renal function have demonstrated an association between the two. However, the relationship between PWV and CAC is not well understood in CKD cases where more complex risk factors, such as uremia or altered mineral metabolism, are prevalent. Therefore, we evaluated the association between brachial ankle PWV (baPWV) and CAC in a predialysis CKD cohort of Korean adults.

\section{Patients and Methods}

\section{Study Design and Population}

This is a cross-sectional study designed to examine the association between baPWV and CAC in CKD patients. Study subjects were participants in the prospective Korean Cohort Study for Outcome in Patients With Chronic Kidney Disease (KNOW-CKD). A detailed protocol of this study was published previously [14]. The study protocol was approved by the Institu- 


\section{Kidney \\ Blood Pressure \\ Research}

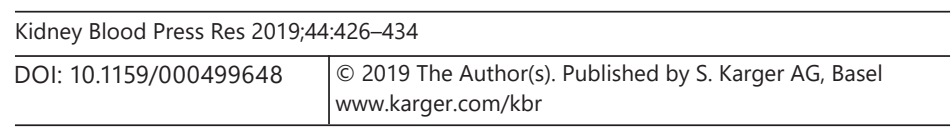

Hyun et al.: Arterial Stiffness and Coronary Calcification

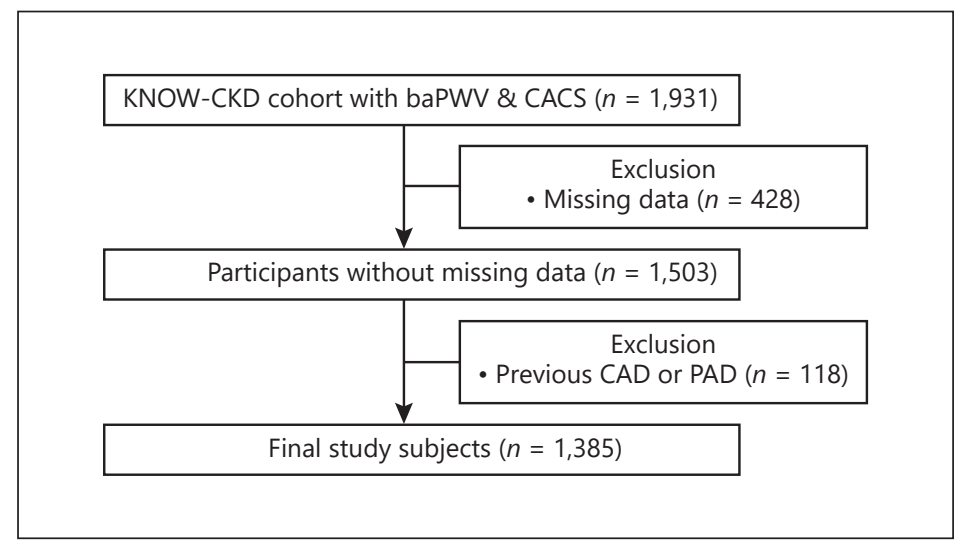

Fig. 1. Algorithm for selecting study subjects from the KNOWCKD cohort. baPWV, brachial-ankle pulse wave velocity; CACS, coronary artery calcium score; CAD, coronary artery disease; PAD, peripheral artery disease.

tional Review Board at each participating clinical center in 2011. In brief, KNOW-CKD is a prospective cohort study that enrolled subjects aged 20-75 years with predialysis CKD stage 1-5. Nine nephrology centers at major university hospitals throughout Korea enrolled 2,238 adults with CKD over a 5-year period from 2011-2015. Participating individuals will be monitored for approximately 10 years until death or until end-stage renal disease occurs. In the entire cohort, 1,931 patients who underwent coronary computed tomography (CT) and baPWV were included in the analysis. We excluded 487 patients with missing data regarding our variables of interest. Additionally, we excluded 118 patients with previous history of coronary artery disease or peripheral artery disease. Finally, 1,385 patients were included in the study (Fig. 1).

\section{Clinical and Laboratory Measurements}

Data regarding socio-demographic information, medical history, medication use, and health-related behaviors were collected through a self-administered questionnaire, with the assistance of trained staff. Anthropometric data and resting blood pressure (BP) were measured by trained nurses. Blood samples were collected after fasting for at least 10 hours. Random urine samples from midstream collection were used to measure the urine protein to creatinine ratio (PCR). Serum creatinine and 25-(OH)-vit D were measured at the central laboratory. Other biochemical analyses were done at the local laboratory of each participating center. Serum creatinine levels were measured using the isotope dilution mass spectroscopy (IDMS)-traceable method. eGFR was calculated using the Chronic Kidney Disease Epidemiology Collaboration (CKD-EPI) equation [15]: eGFR $=141 \times \min$ (serum creatinine/ $\kappa, 1)^{\alpha} \times \max (\text { serum creatinine } / \kappa, 1)^{-1.209} \times 0.993^{\text {age }} \times 1.018$ (if female) $\times 1.159$ (if black), where $\kappa=0.7$ for females and 0.9 for males, $\alpha=-0.329$ for females and -0.411 for males, min indicates the minimum of serum creatinine/ $\kappa$ or 1 , and max indicates the maximum of serum creatinine/ $\kappa$ or 1 . Hypertension was defined as systolic blood pressure $>140 \mathrm{~mm} \mathrm{Hg}$, diastolic blood pressure $>90 \mathrm{~mm} \mathrm{Hg}$, or a history of hypertension. Diabetes mellitus was defined as a fasting serum glucose $>126 \mathrm{mg} / \mathrm{dL}$ or a history of diabetes or anti-diabetic treatment.

\section{baPWV Measurement}

baPWV was measured using automated waveform analyzers VP-1000, VP-1000 plus or VP-2000 (OMRON, Kyoto, Japan) according to the manufacturer's protocol. The PWV between the bilateral brachial arteries and the ankle was measured by placing both arms and the ankle in a cuff, to which an oscillometric sensor was implanted. The mean of the right and left baPWV was used to assess arterial stiffness in our analyses. 
Table 1. Baseline characteristics in relation to baPWV

\begin{tabular}{|c|c|c|c|c|c|c|}
\hline \multirow[t]{2}{*}{ Characteristics } & \multirow[t]{2}{*}{ Total } & \multicolumn{4}{|l|}{ baPWV quartiles } & \multirow{2}{*}{$\begin{array}{l}p \text { for } \\
\text { trend }\end{array}$} \\
\hline & & 1 & 2 & 3 & 4 & \\
\hline$N$ & 1,385 & 346 & 346 & 346 & 347 & \\
\hline $\mathrm{PWV}, \mathrm{cm} / \mathrm{s}$ & $1,521.3 \pm 333.9$ & $1,179.8 \pm 81.5$ & $1,366.4 \pm 43.7$ & $1,556.2 \pm 72.5$ & $1,981.4 \pm 279.5$ & $<0.001$ \\
\hline CACS & $0.0(0.0-64.8)$ & $0.0(0.0-0.7)$ & $0.0(0.0-9.2)$ & $3.9(0.0-92)$ & $62.0(1.3-391.9)$ & $<0.001$ \\
\hline CAC & $290(20.9)$ & $22(6.4)$ & $34(9.8)$ & $82(23.7)$ & $152(43.8)$ & $<0.001$ \\
\hline Age, years & $52.6 \pm 12.3$ & $43.7 \pm 11.1$ & $49.7 \pm 10.9$ & $55.3 \pm 10.4$ & $61.9 \pm 8.6$ & $<0.001$ \\
\hline Male sex & $815(58.8)$ & $193(55.8)$ & $209(60.4)$ & $201(58.1)$ & $212(61.1)$ & 0.249 \\
\hline BMI, $\mathrm{kg} / \mathrm{m}^{2}$ & $24.5 \pm 3.5$ & $24.5 \pm 3.9$ & $24.4 \pm 3.6$ & $24.5 \pm 3.3$ & $24.7 \pm 3.2$ & 0.371 \\
\hline WHR & $0.90 \pm 0.07$ & $0.88 \pm 0.06$ & $0.89 \pm 0.07$ & $0.90 \pm 0.06$ & $0.92 \pm 0.06$ & $<0.001$ \\
\hline Systolic BP, mm Hg & $128 \pm 16$ & $121 \pm 13$ & $126 \pm 14$ & $129 \pm 15$ & $135 \pm 18$ & $<0.001$ \\
\hline Diastolic BP, mm Hg & $77 \pm 11$ & $75 \pm 10$ & $78 \pm 10$ & $78 \pm 12$ & $77 \pm 12$ & 0.040 \\
\hline Glucose, mg/dL & $110 \pm 39$ & $101 \pm 30$ & $105 \pm 34$ & $111 \pm 36$ & $122 \pm 50$ & $<0.001$ \\
\hline $\mathrm{TC}, \mathrm{mg} / \mathrm{dL}$ & $177 \pm 38$ & $179 \pm 37$ & $179 \pm 38$ & $176 \pm 39$ & $172 \pm 38$ & 0.014 \\
\hline LDL cholesterol, mg/dL & $99 \pm 31$ & $102 \pm 32$ & $101 \pm 32$ & $99 \pm 30$ & $95 \pm 30$ & 0.003 \\
\hline hsCRP, mg/dL & $0.6(0.2-1.7)$ & $0.5(0.2-1.6)$ & $0.5(0.2-1.4)$ & $0.7(0.3-1.7)$ & $0.9(0.3-2.1)$ & $<0.001$ \\
\hline $\mathrm{eGFR}, \mathrm{mL} / \mathrm{min} / 1.73 \mathrm{~m}^{2}$ & $55.7 \pm 32.2$ & $72.2 \pm 33.9$ & $61.2 \pm 33.0$ & $50.2 \pm 28.0$ & $39.2 \pm 22.8$ & $<0.001$ \\
\hline Calcium, mg/dL & $9.1 \pm 0.5$ & $9.2 \pm 0.5$ & $9.2 \pm 0.5$ & $9.2 \pm 0.5$ & $9.0 \pm 0.6$ & $<0.001$ \\
\hline Phosphorus, mg/dL & $3.7 \pm 0.7$ & $3.6 \pm 0.6$ & $3.6 \pm 0.6$ & $3.7 \pm 0.6$ & $3.9 \pm 0.8$ & $<0.001$ \\
\hline 25-(OH)-vit D, ng/mL & $17.4 \pm 7.4$ & $17.8 \pm 7.6$ & $18.4 \pm 7.4$ & $17 \pm 7.1$ & $16.5 \pm 7.2$ & 0.003 \\
\hline Intact PTH, $\mathrm{pg} / \mathrm{mL}$ & 50 (33-82) & $41(28-65)$ & $48(34-80)$ & $51(35-88)$ & $61(43-105)$ & $<0.001$ \\
\hline Urine PCR, g/g & $0.5(0.2-1.6)$ & $0.4(0.1-0.9)$ & $0.4(0.1-1.1)$ & $0.6(0.2-1.7)$ & $1.0(0.3-2.7)$ & $<0.001$ \\
\hline Hypertension & $1,334(96.3)$ & $309(89.3)$ & $337(97.4)$ & $344(99.4)$ & $344(99.1)$ & $<0.001$ \\
\hline Diabetes & 461 (33.3) & 44 (12.7) & 69 (19.9) & $129(37.3)$ & $219(63.1)$ & $<0.001$ \\
\hline Dyslipidemia & $740(53.5)$ & $222(64.2)$ & $190(54.9)$ & $176(51.0)$ & $152(43.8)$ & $<0.001$ \\
\hline Current smoker & $235(17.0)$ & $66(19.1)$ & $56(16.2)$ & $60(17.3)$ & $53(15.3)$ & 0.256 \\
\hline
\end{tabular}

Values for categorical variables are reported as a number (percentage), continuous variables as mean \pm standard deviation or median (interquartile range). $p$ values are for analysis of variance or Kruskal-Wallis tests for continuous variables and $\chi^{2}$ tests for categorical variables. PWV, pulse wave velocity; CACS, coronary calcium score; CAC, coronary artery calcification; BMI, body mass index; WHR, waist-hip ratio; BP, blood pressure; TC, total cholesterol; LDL, low-density lipoprotein; hsCRP, high-sensitivity C-reactive protein; eGFR, estimated glomerular filtration rate; PTH, parathyroid hormone; PCR, protein to creatinine ratio.

\section{Coronary Artery Calcium Score (CACS) Measurement}

Electrocardiography-gated multislice computed tomography scans of the thorax were performed using a standard protocol. Quantitative CACS was calculated as described by Agatston et al. [16] We defined the presence of CAC as CACS $>100$, which also differentiates between mild and moderate-to-severe coronary artery disease [17-19].

\section{Statistical Analysis}

Participants were divided into four groups according to baPWV quartile. Continuous variables were expressed as mean \pm standard deviation or median and interquartile range. Continuous variables were compared between two groups with a $t$ test or the Mann-Whitney $\mathrm{U}$ test, and continuous variables were compared between the four groups with analysis of variance or the Kruskal-Wallis test. Categorical variables were expressed as percentages, and comparisons between the patient groups were performed with the $\chi^{2}$ test.

To evaluate the associations between serum baPWV categories and CACS, we used a Tobit regression model for natural log (CACS +1 ). We estimated ratios and 95\% confidence intervals (CIs) for CACS + 1 comparing the higher baPWV categories with the lowest baPWV category. We further used logistic regression analysis to estimate the odds ratio (OR) and confidence interval (CI) for the presence of CAC in higher baPWV categories compared with the lowest baPWV category. We then conducted sensitivity analyses using CAC with different cutoff values (CACS $>0$, CACS $>200$, and CACS $>400$ ). Statistical analyses were performed with Stata Version 15.0 (StataCorp LP, College Station, TX, USA). 
Table 2. Multivariate-adjusted CACS ratios according to baPWV

\begin{tabular}{|c|c|c|c|c|c|c|}
\hline \multirow[t]{2}{*}{ baPWV quartile } & \multicolumn{2}{|l|}{ Model 1} & \multicolumn{2}{|l|}{ Model 2} & \multicolumn{2}{|l|}{ Model 3} \\
\hline & Ratios (95\% CI) & $p$ & Ratios (95\% CI) & $p$ & Ratios (95\% CI) & $p$ \\
\hline 1 & Reference & & Reference & & Reference & \\
\hline 2 & $1.29(0.67-2.50)$ & 0.448 & $1.11(0.59-2.09)$ & 0.740 & $1.11(0.59-2.08)$ & 0.745 \\
\hline 3 & 3.91 (1.99-7.68) & $<0.001$ & $2.08(1.08-4.01)$ & 0.029 & $2.03(1.05-3.92)$ & 0.034 \\
\hline 4 & $11.76(5.8-23.84)$ & $<0.001$ & $3.44(1.66-7.14)$ & 0.001 & 3.03 (1.59-6.87) & 0.001 \\
\hline
\end{tabular}

Model 1: Adjusted for age and sex. Model 2: Adjusted for model $1+$ WHR, systolic blood pressure, diabetes, eGFR, LDL cholesterol, hsCRP, urine protein to creatinine ratio, and current smoking. Model 3: Adjusted for model 2 + calcium, phosphorus, 25-OH-vit D, and intact PTH. CACS, coronary artery calcium score; CI, confidence interval; WHR, waist-hip ratio; eGFR, estimated glomerular filtration rate; LDL, low-density lipoprotein; hsCRP, high-sensitivity C-reactive protein; PTH, parathyroid hormone.

Table 3. Multivariate-adjusted ORs (95\% CI) for CAC according to baPWV

\begin{tabular}{|c|c|c|c|c|c|c|}
\hline \multirow[t]{2}{*}{ baPWV quartile } & \multicolumn{2}{|l|}{ Model 1} & \multicolumn{2}{|l|}{ Model 2} & \multicolumn{2}{|l|}{ Model 3} \\
\hline & OR $(95 \%$ CI $)$ & $p$ & OR $(95 \% \mathrm{CI})$ & $p$ & OR (95\% CI) & $p$ \\
\hline 1 & reference & & reference & & reference & \\
\hline 2 & $0.99(0.55-1.78)$ & 0.962 & $0.87(0.47-1.61)$ & 0.664 & $0.87(0.47-1.61)$ & 0.652 \\
\hline 3 & $2.15(1.25-3.68)$ & 0.005 & $1.56(0.88-2.75)$ & 0.128 & $1.55(0.88-2.75)$ & 0.131 \\
\hline 4 & $3.64(2.12-6.26)$ & $<0.001$ & $1.99(1.10-3.60)$ & 0.024 & $1.98(1.09-3.60)$ & 0.025 \\
\hline
\end{tabular}

Model 1: Adjusted for age and sex. Model 2: Adjusted for model $1+$ WHR, systolic blood pressure, diabetes, eGFR, LDL cholesterol, hsCRP, urine protein to creatinine ratio, and current smoking. Model 3: Adjusted for model $2+$ calcium, phosphorus, 25-OH-vit D, and intact PTH. OR, odds ratio; CAC, coronary artery calcification; CI, confidence interval; WHR, waist-hip ratio; eGFR, estimated glomerular filtration rate; LDL, low-density lipoprotein; hsCRP, high-sensitivity C-reactive protein; PTH, parathyroid hormone.

\section{Results}

The baseline characteristics of study participants classified by baPWV quartiles are summarized in Table 1. CAC was more prevalent among the higher baPWV groups $(6.4,9.8$, 23.7 , and $43.8 \%$ for the 1 st to 4 th baPWV quartiles, respectively, $p<0.001$ ). Overall, the higher was the baPWV quartile, the worse was the cardiometabolic profile. For example, patients with higher baPWV were older and had a higher waist-hip ratio, higher systolic BP, lower eGFR, and higher intact parathyroid hormone. Likewise, patients who were male and had diabetes had higher prevalence in the higher baPWV quartiles.

In Tobit regression analysis, high baPWV was associated with higher CACS after multivariate adjustment. The CACS ratio comparing the highest baPWV quartile with the lowest quartile was 3.03 (95\% CI, 1.59-6.87) in the fully adjusted model 3 (Table 2).

Similarly, high baPWV was significantly associated with CAC after adjusting for traditional and non-traditional CV risk factors in multivariate logistic analysis. As shown in Table 3 , the OR for CAC in the highest baPWV quartile compared with the lowest quartile was 1.98 (95\% CI, 1.09-3.60) in the fully adjusted model 3.

For our study, we defined CAC as CACS $>100$, but the associations between baPWV and CAC with different cutoffs were also evaluated in sensitivity analysis (Table 4). Compared with the lowest baPWV quartile patients, the highest quartile patients had a CAC OR of 2.55 
Table 4. Multivariate-adjusted ORs ( $95 \% \mathrm{CI}$ ) for CAC with different cutoffs

\begin{tabular}{|c|c|c|c|c|c|c|}
\hline \multirow[t]{2}{*}{ baPWV quartile } & \multicolumn{2}{|l|}{ CACS >0 } & \multicolumn{2}{|l|}{ CACS $>200$} & \multicolumn{2}{|l|}{ CACS $>400$} \\
\hline & OR $(95 \%$ CI) & $p$ & OR $(95 \% \mathrm{CI})$ & $p$ & OR $(95 \% \mathrm{CI})$ & $p$ \\
\hline 1 & reference & & reference & & reference & \\
\hline 2 & $1.09(0.74-1.61)$ & 0.647 & $0.81(0.38-1.72)$ & 0.583 & $0.77(0.29-2.05)$ & 0.600 \\
\hline 3 & $1.30(0.87-1.94)$ & 0.206 & $1.37(0.69-2.73)$ & 0.364 & $1.57(0.67-3.71)$ & 0.300 \\
\hline 4 & $1.65(1.02-2.65)$ & 0.040 & $2.13(1.06-4.29)$ & 0.034 & $2.55(1.08-6.04)$ & 0.033 \\
\hline
\end{tabular}

Adjusted for age, sex, WHR, systolic blood pressure, diabetes, eGFR, LDL cholesterol, hsCRP, urine protein to creatinine ratio, current smoking, calcium, phosphorus, 25-OH-vit D, and intact PTH. OR, odds ratio; CAC; coronary artery calcification; CACS, coronary artery calcium score; CI, confidence interval; WHR, waist-hip ratio; eGFR, estimated glomerular filtration rate; LDL, low-density lipoprotein; hsCRP, high-sensitivity C-reactive protein; PTH, parathyroid hormone.

(95\% CI, 1.08-6.04; $p<0.001$ ) when CAC was defined as CACS $>400$. The results were similar when CAC was defined as CACS $>0$ or CACS $>200$. In further sensitivity analysis, the association between baPWV and CAC was similar across clinically relevant subgroups. There were no significant interactions by sex (female vs. male, $p$ for interaction $=0.110$ ), age ( $<50 \mathrm{vs.} \geq 50$ years, $p$ for interaction $=0.115)$, BMI $\left(<23.5 \mathrm{vs} . \geq 23.5 \mathrm{~kg} / \mathrm{m}^{2}, p\right.$ for interaction $\left.=0.816\right)$, diabetes (no vs. yes, $p$ for interaction $=0.595$ ), eGFR $\left(<45 \mathrm{vs.} \geq 45 \mathrm{ml} / \mathrm{min} / 1.72 \mathrm{~m}^{2}, p\right.$ for interaction $=0.457)$, proteinuria ( $<1.0$ vs. $\geq 1.0 \mathrm{~g} /$ day, $p$ for interaction $=0.295)$, or hsCRP $(<1.0$ vs. $\geq 1.0 \mathrm{mg} / \mathrm{dL}, p$ for interaction $=0.278)$.

\section{Discussion}

In this study, we found that high baPWV was associated with CAC in predialysis CKD patients, and that this relationship was independent of traditional and non-traditional CV risk factors. Furthermore, this association was preserved with different CAC cutoff values and was consistent across different subgroup analyses.

These results are similar to those of previous studies of patients with preserved renal function. Torii et al. analyzed randomly selected Japanese men $(n=986)$ aged $40-79$ years and they showed that elevated baPWV was associated with higher CAC prevalence in the general population [20]. They found that the optimal baPWV cutoff level to detect CAC was $1,612 \mathrm{~cm} / \mathrm{s}$. Similarly, in an international study of 1,131 healthy middle-aged men, baPWV was cross-sectionally associated with CAC, and the odds for developing CAC increased by $19 \%$ per every $100-\mathrm{cm} / \mathrm{s}$ increase in baPWV [21]. The largest study in this regard was the study by Cainzos-Achirica et al. In a single-center study of young and middle-aged Korean patients $(n=15,185)$, baPWV was independently associated with CAC presence and severity in asymptomatic adults [22]. We also found that the addition of baPWV to traditional risk factors significantly improved CAC diagnosis. Furthermore, another study by our research group found that high baPWV was also associated with CAC progression [23].

Several studies have evaluated the association between arterial stiffness and CAC in CKD patients. Haydar et al. analyzed 55 hemodialysis patients [24] and found that aortic PWV correlated with total CAC after correcting for age, dialysis duration, and timeaveraged C-reactive protein. In another study of 104 predialysis patients by Toussaint et al., carotid-femoral PWV (cfPWV) correlated with coronary calcium score and calcium volume [25]. Interestingly, in a study of 132 hemodialysis patients by Di Iorio et al., multivariate analyses revealed that CAC progression was associated with a significant increase 


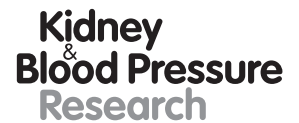

Kidney
Blood Pressure
Research \begin{tabular}{l|l}
\hline Kidney Blood Press Res 2019;44:426-434 \\
\hline DOI: 10.1159/000499648 & $\begin{array}{l}\text { @ 2019 The Author(s). Published by S. Karger AG, Basel } \\
\text { www.karger.com/kbr }\end{array}$ \\
\hline
\end{tabular}

Hyun et al.: Arterial Stiffness and Coronary Calcification

in cfPWV [26]. However, previous studies of CKD patients are very small and do not take into account important risk factors such as sex, obesity, renal function, or phosphorus level. To the best of our knowledge, this is the only large-scale study of CKD patients that has identified a relationship between arterial stiffness and CAC after adjusting for various risk factors.

There are several candidate mechanisms underlying the association between arterial stiffness and CAC. First, arterial stiffness is not independent of vascular calcification and may be a physiologic expression of arteriosclerosis that is an outcome of medial and intimal calcification [27]. In CAC cases, both intimal and medial calcifications are observed [28], although intimal calcification is more common than vascular calcification in CKD cases where medial calcification is more prevalent [29]. Second, arterial stiffness can induce atherosclerosis directly. Intimal calcification is an important finding as atherosclerosis progresses and atherosclerotic plaques form [30,31]. Arterial wall shear stress, endothelial dysfunction, and alterations in the extracellular matrix, such as elastin fragmentation or collagen deposition combined with high arterial stiffness, are suggested mechanisms underlying atherogenesis [32]. Third, arterial stiffness and vascular calcification share risk factors including age, sex, blood pressure, diabetes, inflammation, and chronic kidney disease, which are the basis for linking arterial stiffness to vascular calcification [30, 32]. We have controlled for these risk factors to look for independent relationships between the two, but it is also possible that there are residual or unmeasured confounders.

As mentioned above, recent studies have found that subclinical atherosclerosis detected as CAC is a predictor of CVD and high CV mortality in CKD patients as well as in the general population $[12,13]$. However, coronary calcium scoring requires high costs, skilled expertise, and risk of radiation exposure. In contrast, PWV measurement is relatively simple, cost effective, and does not require a separate expert to read the results. Furthermore, baPWV does not require the inguinal area to be exposed or special training to take measurements compared with cfPWV [33,34]. In this regard, this study offers clinical implications for increased significance of baPWV as a CV risk stratification tool in CKD patients.

Our results should be interpreted with caution due to a few limitations. First, this study is cross-sectional in design. We cannot define a causal relationship between arterial stiffness and CAC. Second, some information that might improve our analysis was not available, such as physical activity and markers of insulin resistance. Despite these limitations, the relatively large number of study subjects from different centers, our well-structured research design, and our inclusion of various risk factors in CKD are strengths of this study.

In conclusion, increased arterial stiffness measured by high baPWV was associated with CAC in a predialysis CKD cohort of Korean adults. These results remained significant after adjusting for various traditional and non-traditional CV risk factors and after using different cutoff values for CAC or different subgroup rearrangements. However, this crosssectional study alone cannot elucidate a specific underlying mechanism for this association. Further research is warranted to determine the exact mechanism by which arterial stiffness leads to the development of CAC and CVD in CKD patients. Longitudinal studies may help determine the role of arterial stiffness in the development or progression of CAC in CKD.

\section{Disclosure Statement}

No conflict of interest to declare. 


\section{Kidney \\ Blood Pressure Research}

\begin{tabular}{l|l}
\hline Kidney Blood Press Res 2019;44:426-434 \\
\hline DOI: 10.1159/000499648 & $\begin{array}{l}\text { @ 2019 The Author(s). Published by S. Karger AG, Basel } \\
\text { www.karger.com/kbr }\end{array}$ \\
\hline
\end{tabular}

Hyun et al.: Arterial Stiffness and Coronary Calcification

\section{Funding Sources}

This work was supported by the Research Program funded by the Korea Centers for Disease Control and Prevention (2011E3300300, 2012E3301100, 2013E3301600, 2013E3301601, 2013E3301602, 2016E3300200, 2016E3300201).

\section{Author Contributions}

Young Youl Hyun: Drafting the article, analysis and interpretation of data, revising the article and final approval of the article.

Hyang Kim, Kook-Hwan Oh, Curie Ahn, Sue K. Park, Dong Wan Chae, Seung Hyeok Han, Yong-Soo Kim, Sung Woo Lee, Chang Seong Kim: Analysis and interpretation of data, revising the article and final approval of the article.

Kyu-Beck Lee: Conception or design, analysis and interpretation of data and final approval of the article.

\section{References}

1 Tonelli M, Wiebe N, Culleton B, House A, Rabbat C, Fok M, et al. Chronic kidney disease and mortality risk: a systematic review. J Am Soc Nephrol. 2006 Jul;17(7):2034-47.

2 Michel Safar MO. Principles and definitions of arterial stiffness, wave reflections and pulse pressure amplification: Handbook of Hypertension. Volume 23. Amsterdam: Elsevier; 2006. pp. 3-20.

3 Briet M, Bozec E, Laurent S, Fassot C, London GM, Jacquot C, et al. Arterial stiffness and enlargement in mildto-moderate chronic kidney disease. Kidney Int. 2006 Jan;69(2):350-7.

4 Chue CD, Townend JN, Steeds RP, Ferro CJ. Arterial stiffness in chronic kidney disease: causes and consequences. Heart. 2010 Jun;96(11):817-23.

5 Georgianos PI, Sarafidis PA, Liakopoulos V. Arterial Stiffness: A Novel Risk Factor for Kidney Injury Progression? Am J Hypertens. 2015 Aug;28(8):958-65.

6 Zoungas S, Cameron JD, Kerr PG, Wolfe R, Muske C, McNeil JJ, et al. Association of carotid intima-medial thickness and indices of arterial stiffness with cardiovascular disease outcomes in CKD. Am J Kidney Dis. 2007 Oct;50(4):622-30.

7 Pannier B, Guérin AP, Marchais SJ, Safar ME, London GM. Stiffness of capacitive and conduit arteries: prognostic significance for end-stage renal disease patients. Hypertension. 2005 Apr;45(4):592-6.

8 Bover J, Evenepoel P, Ureña-Torres P, Vervloet MG, Brandenburg V, Mazzaferro S, et al.; CKD-MBD Working Group of ERA-EDTA. Pro: cardiovascular calcifications are clinically relevant. Nephrol Dial Transplant. 2015 Mar;30(3):345-51.

9 Bellasi A, Raggi P. Vascular imaging in chronic kidney disease. Curr Opin Nephrol Hypertens. 2012 Jul;21(4): 382-8.

10 Nigwekar SU, Solid CA, Ankers E, Malhotra R, Eggert W, Turchin A, et al. Quantifying a rare disease in administrative data: the example of calciphylaxis. J Gen Intern Med. 2014 Aug;29(S3 Suppl 3):S724-31.

11 Greenland P, Alpert JS, Beller GA, Benjamin EJ, Budoff MJ, Fayad ZA, et al.; American College of Cardiology Foundation; American Heart Association. 2010 ACCF/AHA guideline for assessment of cardiovascular risk in asymptomatic adults: a report of the American College of Cardiology Foundation/American Heart Association Task Force on Practice Guidelines. J Am Coll Cardiol. 2010 Dec;56(25):e50-103.

12 Matsushita K, Sang Y, Ballew SH, Shlipak M, Katz R, Rosas SE, et al. Subclinical atherosclerosis measures for cardiovascular prediction in CKD. J Am Soc Nephrol. 2015 Feb;26(2):439-47.

13 Chen J, Budoff MJ, Reilly MP, Yang W, Rosas SE, Rahman M, et al.; CRIC Investigators. Coronary Artery Calcification and Risk of Cardiovascular Disease and Death Among Patients With Chronic Kidney Disease. JAMA Cardiol. 2017 Jun;2(6):635-43.

14 Oh KH, Park SK, Park HC, Chin HJ, Chae DW, Choi KH, et al.; Representing KNOW-CKD Study Group. KNOW-CKD (KoreaN cohort study for Outcome in patients With Chronic Kidney Disease): design and methods. BMC Nephrol. 2014 May;15(1):80.

15 Levey AS, Stevens LA, Schmid CH, Zhang YL, Castro AF 3rd, Feldman HI, et al.; CKD-EPI (Chronic Kidney Disease Epidemiology Collaboration). A new equation to estimate glomerular filtration rate. Ann Intern Med. 2009 May;150(9):604-12. 
16 Agatston AS, Janowitz WR, Hildner FJ, Zusmer NR, Viamonte M Jr, Detrano R. Quantification of coronary artery calcium using ultrafast computed tomography. J Am Coll Cardiol. 1990 Mar;15(4):827-32.

17 Raggi P, Callister TQ, Cooil B, He ZX, Lippolis NJ, Russo DJ, et al. Identification of patients at increased risk of first unheralded acute myocardial infarction by electron-beam computed tomography. Circulation. 2000 Feb; 101(8):850-5.

18 Berman DS, Wong ND, Gransar H, Miranda-Peats R, Dahlbeck J, Hayes SW, et al. Relationship between stressinduced myocardial ischemia and atherosclerosis measured by coronary calcium tomography. J Am Coll Cardiol. 2004 Aug;44(4):923-30.

19 Pletcher MJ, Tice JA, Pignone M, Browner WS. Using the coronary artery calcium score to predict coronary heart disease events: a systematic review and meta-analysis. Arch Intern Med. 2004 Jun;164(12):1285-92.

20 Torii S, Arima H, Ohkubo T, Fujiyoshi A, Kadota A, Takashima N, et al.; SESSA Research Group. Association between Pulse Wave Velocity and Coronary Artery Calcification in Japanese men. J Atheroscler Thromb. 2015; 22(12):1266-77.

21 Vishnu A, Choo J, Wilcox B, Hisamatsu T, Barinas-Mitchell EJ, Fujiyoshi A, et al.; ERA JUMP Study Group. Brachial-ankle pulse wave velocity is associated with coronary calcification among 1131 healthy middle-aged men. Int J Cardiol. 2015;189:67-72.

22 Cainzos-Achirica M, Rampal S, Chang Y, Ryu S, Zhang Y, Zhao D, et al. Brachial-ankle pulse wave velocity is associated with coronary calcium in young and middle-aged asymptomatic adults: The Kangbuk Samsung Health Study. Atherosclerosis. 2015 Aug;241(2):350-6.

23 Lee JY, Ryu S, Lee SH, Kim BJ, Kim BS, Kang JH, et al. Association between brachial-ankle pulse wave velocity and progression of coronary artery calcium: a prospective cohort study. Cardiovasc Diabetol. 2015 Nov;14(1): 147.

24 Haydar AA, Covic A, Colhoun H, Rubens M, Goldsmith DJ. Coronary artery calcification and aortic pulse wave velocity in chronic kidney disease patients. Kidney Int. 2004 May;65(5):1790-4.

25 Lemos MM, Jancikic AD, Sanches FM, Christofalo DM, Ajzen SA, Miname MH, et al. Pulse wave velocity-a useful tool for cardiovascular surveillance in pre-dialysis patients. Nephrol Dial Transplant. 2007 Dec;22(12): 3527-32.

26 Di Iorio B, Nargi O, Cucciniello E, Bellizzi V, Torraca S, Russo D, et al.; INDEPENDENT study investigators. Coronary artery calcification progression is associated with arterial stiffness and cardiac repolarization deterioration in hemodialysis patients. Kidney Blood Press Res. 2011;34(3):180-7.

27 Tölle M, Reshetnik A, Schuchardt M, Höhne M, van der Giet M. Arteriosclerosis and vascular calcification: causes, clinical assessment and therapy. Eur J Clin Invest. 2015 Sep;45(9):976-85.

28 Nakamura S, Ishibashi-Ueda H, Niizuma S, Yoshihara F, Horio T, Kawano Y. Coronary calcification in patients with chronic kidney disease and coronary artery disease. Clin J Am Soc Nephrol. 2009 Dec;4(12):1892-900.

29 Shroff R, Long DA, Shanahan C. Mechanistic insights into vascular calcification in CKD. J Am Soc Nephrol. 2013 Feb;24(2):179-89.

30 Doherty TM, Asotra K, Fitzpatrick LA, Qiao JH, Wilkin DJ, Detrano RC, et al. Calcification in atherosclerosis: bone biology and chronic inflammation at the arterial crossroads. Proc Natl Acad Sci USA. 2003 Sep;100(20): 11201-6.

31 Vervloet M, Cozzolino M. Vascular calcification in chronic kidney disease: different bricks in the wall? Kidney Int. 2017 Apr;91(4):808-17.

32 Palombo C, Kozakova M. Arterial stiffness, atherosclerosis and cardiovascular risk: pathophysiologic mechanisms and emerging clinical indications. Vascul Pharmacol. 2016 Feb;77:1-7.

33 Sugawara J, Tanaka H. Brachial-Ankle Pulse Wave Velocity: Myths, Misconceptions, and Realities. Pulse (Basel). 2015 Sep;3(2):106-13.

34 Townsend RR, Wilkinson IB, Schiffrin EL, Avolio AP, Chirinos JA, Cockcroft JR, et al.; American Heart Association Council on Hypertension. Recommendations for Improving and Standardizing Vascular Research on Arterial Stiffness: A Scientific Statement From the American Heart Association. Hypertension. 2015 Sep;66(3): 698-722. 